\title{
Etat de la Radiologie Dans les Chu de Bangui et de Bimbo, Centrafrique
}

\section{Kouandongui Bangue Songrou Francky, Bidan Tapiade Euloge,}

Centre Hospitalier Universitaire Maman Elisabeth Domitien De Bimbo,

République Centrafrique

\author{
Ouimon Moise,
}

Mobima Timothée,

Hôpital De L'amitié De Bangui, République Centrafrique

\section{Résumé}

Le but de l'étude était de déterminer le profil du personnel de radiologie exerçant dans les principales structures sanitaires de Centrafrique et d'y répertorier toutes les installations. C'était étude descriptive réalisée entre le mois d'octobre et le mois de novembre 2018 sous forme d'enquête. L'ensemble du personnel était constitué de 18 praticiens, tous de sexe masculin, avec un moyen de 47,55 ans. La durée moyenne d'années d'expérience était de 12,77ans (extrêmes de 2 à 33 ans). Le personnel était composé de médecins radiologues $(\mathrm{n}=4 ; 22,2 \%)$, des techniciens supérieurs en radiologie et imagerie médicale $(n=7 ; 38,9 \%)$, des infirmiers diplômés d'Etat (IDE), ( $\mathrm{n}=3 ; 16,7 \%)$ et des assistants de santé (AS), $(\mathrm{n}=4 ; 22,2 \%)$ Il n'existait aucun personnel en matière de radioprotection et de maintenance des installations radiologiques. Le parc radiologique était constitué exclusivement de la radiographie standard et de l'échographie. L'IRM et la TDM ne comptaient pas parmi les installations radiologiques. Le personnel de radiologie et le parc radiologique de Centrafrique ne répondent pas encore aux exigences de la médecine moderne.

Mots-clés: Personnel, Installation radiologique, Structures sanitaires, Centrafrique 


\title{
Status of Radiology in Bangui and Bimbo Hospitals in Central Africa
}

\section{Kouandongui Bangue Songrou Francky, Bidan Tapiade Euloge,}

Centre Hospitalier Universitaire Maman Elisabeth Domitien De Bimbo,

République Centrafrique

Ouimon Moise,

Mobima Timothée,

Hôpital De L'amitié De Bangui, République Centrafrique

\begin{abstract}
This paper focuses on describing the vocational qualification of radiology staff and all radiological facility in major hospitals in Central Africa Republic. It was a descriptive study from October to November 2018 with 18 practitioners (male). The middle age was 47.55 years while the middle years of experience was 12.77 years. The staff's profile revealed physicians radiologists $(n=4 ; 22.2 \%)$, technicians in radiology and medical imaging ( $\mathrm{n}$ $=7 ; 38.9 \%)$, graduate nurses of the State $(n=3 ; 16.7 \%)$, and helpers of health $(\mathrm{n}=4 ; 22.2 \%)$. There were no personnel for radiation protection and maintenance of radiological facilities. The X-ray was composed exclusively of standard radiography and ultrasound. MRI and CT were not included in the radiological facilities. The radiology staff and the radiological park of Central Africa have not yet met the requirements of modern medicine.
\end{abstract}

Keywords: Staff, Radiological facility, Health structures, Central Africa Republic

\section{Introduction}

L'imagerie médicale est une spécialité pivot, incontournable dans l'exercice de la médecine moderne (FNMR, 2017). En 2018, la plus part des pays africains au Sud du Sahara dispose déjà des équipements modernes de la Radiologie et de l'Imagerie Médicale et un personnel médical qualifié dans ce domaine (Werner, 2010 ; Ongolo-Zogo, 2013). En Centrafrique, aucune étude relative au parc radiologique existant et au profil du personnel exerçant dans le domaine de la Radiologie et de l'Imagerie Médicale, n'a jamais été réalisée. L'objectif de ce travail est d'étudier le profil du personnel de radiologie exerçant dans les principaux établissements sanitaires de 
Centrafrique, de répertorier les installations radiologiques disponibles dans ces structures sanitaires face aux exigences de la médecine moderne. Les Centres Hospitaliers et Universitaires (CHU) de Bangui, capitale de la Centrafrique et de Bimbo, ville la plus proche de Bangui, ont été choisis pour cette étude. Dans ces deux villes, se trouvent tous les médecins spécialistes du pays.

\section{Matériel et méthodes}

Il s'agissait d' une étude descriptive réalisée entre le mois d'octobre et le mois de novembre 2018 sous forme d'enquête auprès des praticiens de radiologie des Centres Hospitaliers et Universitaires de Bangui et de Bimbo, à savoir , Hôpital Communautaire de Bangui (HC), Hôpital de l'Amitié de Bangui (HA), Centre National Hospitalier et Universitaire de Bangui (CNHUB), Centre Hospitalier et Universitaire Maman Elisabeth DOMITIEN de Bimbo (CHUMED. Le matériel utilisé était constitué de fiches de questionnaires dont la première partie était dédiée à l'étude des profils du personnel (l'âge, le sexe, la qualification professionnelle, l'établissement d'appartenance, les années d'expérience) et à la présence d'un personnel réfèrent en maintenance des installations radiologiques et en radioprotection. Cette fiche était remise en mains propres aux différents praticiens après consentement éclairé ; elles ont été récupérées une semaine après. La deuxième partie était consacrée au recensement des différents matériels de radiologie en état marche ou en état de panne ou indisponible (radiographie standard, échographie polyvalente, mammographie, scanner, IRM) dans les CHU visités.

\section{Résultats}

\section{Etat du personnel dans les CHU}

$\mathrm{Au}$ total 18 praticiens travaillent dans les principales structures sanitaires de Centrafrique. La répartition de ce personnel selon le profil a révélé la présence du personnel qualifié, constitué de médecins radiologues $(\mathrm{n}=4 ; 22,2 \%)$, des techniciens supérieurs en radiologie et imagerie médicale $(\mathrm{n}=7 ; 38,9 \%)$. D'autres praticiens non qualifiés (en radiologie et imagerie médicale) dont les infirmiers diplômés d'Etat (IDE) et les assistants de santé (AS), tous formés à la technique de réalisation des examens radiographiques participaient aussi aux activités des services de radiologie à hauteur respective de $16,7 \%(\mathrm{n}=3)$ et de $22,2 \%(\mathrm{n}=4)$. Il n'existait aucun personnel référent en matière de radioprotection et de maintenance des installations radiologiques dans tous les établissements visités. Le Tableau I décrit le profil du personnel exerçant dans les principales structures sanitaires de Bangui et de Bimbo. 
Tableau I. Répartition des praticiens par profil dans les CHU de Bangui et de Bangui (Source hospitalière)

\begin{tabular}{lcc}
\hline & Effectif & Pourcentage \\
\hline Radiologues & 4 & 22 \\
Techniciens en radiologie & 7 & 39 \\
IDE formés & 3 & 17 \\
AS formés & 4 & 22 \\
Maintenancier en radiologie & 0 & 0 \\
Référent en radioprotection & 0 & 0 \\
\hline Total & $\mathbf{1 8}$ & $\mathbf{1 0 0}$ \\
\hline
\end{tabular}

L'ensemble de ce personnel était reparti dans quatre Centres Hospitaliers et Universitaires dont trois à Bangui et un à Bimbo, Tableau II.

Tableau II. Répartition des praticiens par profil dans les CHU de Bangui et de Bangui (Source hospitalière)

\begin{tabular}{lccccc}
\hline & HA & HC & CNHUB & CHUMED & Total \\
\hline Radiologue & 2 & 1 & 0 & 1 & $\mathbf{4}$ \\
Technicien en radiologie & 2 & 3 & 1 & 1 & $\mathbf{7}$ \\
IDE formés & 1 & 0 & 1 & 1 & $\mathbf{3}$ \\
AS formés & 0 & 1 & 1 & 2 & $\mathbf{4}$ \\
Maintenancier en radiologie & 0 & 0 & 0 & 0 & $\mathbf{0}$ \\
Référent en radioprotection & 0 & 0 & 0 & 0 & $\mathbf{0}$ \\
\hline Total & $\mathbf{5}$ & $\mathbf{5}$ & $\mathbf{3}$ & $\mathbf{5}$ & $\mathbf{1 8}$ \\
\hline
\end{tabular}

L'ensemble des praticiens était de sexe masculin, d'âge moyen de 47,55 ans avec les extrêmes de 31 à 63 ans. La durée moyenne d'années d'expérience était de 12,77ans avec les extrêmes de 2 à 33ans.

Sur les dix-huit praticiens répertoriés, le plus grand effectif se trouvait dans les CHU de l'Hôpital de l'Amitié, de l'hôpital Communautaire et du Centre Hospitalier et Universitaire de Bimbo à hauteur de 5 praticiens $(27,8 \%)$.

\section{Etat du matériel de la Radiologie et de l'Imagerie Médicale}

Le parc radiologique de la Bangui, capitale de la Centrafrique et de Bimbo était constitué principalement de la radiographie standard et de l'échographie. La quasi-totalité des établissements disposaient d'une table ospoumon ( $\mathrm{n}=3$ soit $75 \%$ ) ce qui n'était pas le cas avec la table télécommandée qui n'était disponible que dans un seul établissement à savoir le CHUMED, $(\mathrm{n}=1$ soit $25 \%)$ mais en panne. Un seul établissement $(\mathrm{n}=1$ soit $25 \%)$ disposait d'un système de développement numérique. Un seul établissement disposait d'une mammographie, mais non fonctionnelle depuis la livraison (défaut de fabrication). L'échographe polyvalent était disponible dans tous établissements visités, mais en panne à l'hôpital Communautaire. Les appareils de TDM et d'IRM ne figuraient pas parmi les installations radiologiques disponibles en Centrafrique. Le Tableau III décrit l'état de 
disponibilité et de fonctionnement des équipements de radiologie de Centrafrique (Bimbo et Bangui).

Tableau III. Etat de disponibilité et de fonctionnement des équipements de radiologie existants dans les CHU de Bangui et de Bimbo (Source hospitalière)

\begin{tabular}{|c|c|c|c|c|}
\hline & HA & $\mathrm{HC}$ & CNHUB & CHUMED \\
\hline Radiographie standard & Marche & Marche & Non disponible & Marche \\
\hline Système numérique & Marche & Non disponible & Non disponible & Non disponible \\
\hline Mammographie & $\begin{array}{l}\text { Non } \\
\text { disponible }\end{array}$ & Non disponible & Non disponible & En panne \\
\hline Echographe polyvalent & Marche & En marche & Marche & Marche \\
\hline Scanner & $\begin{array}{l}\text { Non } \\
\text { disponible }\end{array}$ & Non disponible & Non disponible & Non disponible \\
\hline IRM & $\begin{array}{l}\text { Non } \\
\text { disponible }\end{array}$ & Non disponible & Non disponible & Non disponible \\
\hline
\end{tabular}

\section{Discussion}

A l'instar des pays en développement, la République Centrafricaine est confrontée à de graves problèmes de manque de structures, d'équipements, de ressources, voire de personnel qualifié ; et la santé, secteur vital n'est pas épargnée (Bosembo Ilondjo, 2005).

\section{Profil du personnel}

La Centrafrique dispose d'une faculté de sciences de la santé qui forme les médecins généralistes, quelques médecins spécialistes (gynécologues, pédiatres, chirurgiens généralistes) et les paramédicaux (infirmiers diplômés d'Etat, techniciens supérieurs en anesthésie et réanimation, les sages-femmes et assistants de santé). Par contre contrairement à plusieurs pays de la sousrégion (Ongolo-Zogo, 2013 ; Tchaou, 2018 ; Adeyekun, 2010), la filière de la Radiologie et de l'Imagerie Médicale n'est pas encore prise en compte en matière de formation. L'absence d'une telle école de formation peut expliquer en partie cette pénurie en personnels qualifiés au sein des services de Radiologie et d'Imagerie Médicale des CHU et la présence des personnels para médicaux non qualifiés. Tout le personnel qualifié en Radiologie et Imagerie Médicale (radiologues et techniciens supérieurs) est formé à l'étranger. Si la présence des radiologues et des techniciens en Imagerie Médicale est indispensable dans un service de radiologie pour la validation, la réalisation et l'interprétation des examens (Etard, 2010), la disponibilité d'un technicien de maintenance dans un service de Radiologie et d'Imagerie Médicale l'est aussi au même titre. En effet, le maintien du plateau technique dans un état de fonctionnalité permet d'assurer la pérennité des examens radiologiques. Selon Nko'o et al. (2009), l'entretien et l'installation des équipements constituent de véritables défis pour les pays en voie de développement. La présence d'un personnel référent en radioprotection au sein d'un service de radiologie est 
aussi indispensable, elle contribuera à l'application des principes de la radioprotection qui est une obligation réglementaire selon l'Agence Internationale de l'Energie Atomique (AIEA, 2010). La radioprotection vise à mettre les praticiens, les patients et l'environnement à l'abri des effets néfastes des rayonnements ionisants.

\section{Etat du matériel de la Radiologie et de l'Imagerie Médicale}

L'influence de l'imagerie médicale dans la médecine moderne est considérable et s'étend du diagnostic, à la prévention en passant par le suivi des traitements, la pharmacologie sans oublier la recherche médicale en termes de compréhension des maladies (Decouelaere, 2005; FNMR, 2017). La modernisation du plateau technique est un véritable défi en Afrique Sub Saharienne, elle est très coûteuse certes mais elle peut être aussi une source d'économie. Un diagnostic précis et rapide permet une orientation appropriée et immédiate des patients dans leur parcours de soins évitant ainsi des actes médicaux et des hospitalisations inutiles. Elle va permettre aussi d'éviter des évacuations sanitaires, très couteuses. Plusieurs pays d'Afrique Sub Saharienne ont déjà progressé vers la modernisation de leur plateau technique (Werner, 2010). L'état actuel de l'Imagerie médicale à Bangui et à Bimbo (Centrafrique), est très loin de satisfaire aux exigences de la médecine moderne.

L'imagerie analogique est très contraignante. Elle nécessite un traitement chimique pour le développement de l'image sur film analogique (seul moyen de reprographie, d'archivage et unique support pour l'interprétation des images). La qualité de l'image est tributaire de la qualité des bains qui est elle-même est très sensible aux variations climatiques (Werner, 2009). L'imagerie numérique présente l'avantage de pouvoir traiter et améliorer l'image avant impression, avec comme conséquence pas ou peu de rebuts de films (Werner, 2010 ; Gbande, 2017). La mise en place d'un réseau de radiologie numérique sera donc importante, elle pourrait permettre un échange de données d'imagerie médicale entre établissements de manière à permettre un diagnostic à distance et plus rapide des images sans devoir nécessairement attendre la visite d'un radiologiste sur place (Pérusse, 1999). Le renforcement du parc radiologique de Centrafrique par la numérisation des salles de radiologie conventionnelle est nécessaire pour répondre au besoin de performance.

La non disponibilité de la mammographie est un handicap pour une politique de dépistage systématique des cancers du sein. Cette insuffisance des moyens d'exploration pourrait être responsable du diagnostic tardif des cancers du sein chez la femme (Ngou-Mve-Ngouj, 2009). La mammographie est le seul moyen d'imagerie qui permet le diagnostic des lésions précancéreuses (CNLC, 2007). C'est l'examen de base dans le diagnostic des 
cancers du sein, son efficacité est prouvée et le rapport Coût efficacité est acceptable Elle a une sensibilité et une spécificité élevées (Delaloe, 2012). L'échographie complète la mammographie, mais ne la remplace pas, c'est pourquoi on parle aujourd'hui du couple mammo-échographie.

Le recours aux examens d'imagerie médicale tels que le scanner et l'IRM est de plus en plus fréquent au cours de la prise en charge des patients. Le scanner est un examen très sollicité à tel point que le nombre d'examens réalisés chaque année ne cesse d'augmenter (NCRP, 2009), ce qui explique la disponibilité de cette technologie dans la quasi-totalité des pays africains au sud du Sahara (Kouna Ndouongo, 2007 ; Kaya, 2006 ; Guidah, 2012). L'IRM, modalité d'imagerie non ionisante, permet une bonne discrimination entre tissus (même de densité voisine) et le choix de plans de coupes quelconques. Le contraste entre tissus ainsi que certaines fonctions du corps peuvent être rehaussés par l'emploi de marqueurs. Notons également la possibilité de réaliser des images agiographiques 3D sans injection de produit de contraste contrairement au scanner (Bernard, 1987). L'inaccessibilité à cette technologie en Centrafrique a des répercussions négatives non seulement en termes de la pratique médicale, mais aussi en termes de la formation médicale avec un risque de la baisse de la qualité de formations des étudiants et suscitera moins de vocation pour la radiologie et l'imagerie médicale (Adeyekun, 2018).

\section{Conclusion}

Cette étude menée dans les Centres Hospitaliers et Universitaires de Bangui et de Bimbo, où sont concentrés tous les médecins spécialistes de la Centrafrique souligne les faiblesses du plateau technique et la pénurie du personnel qualifié en radiologie et imagerie médicale. Ces structures qualifiées de référence de niveau 1 dans la pyramide sanitaire de la République Centrafricaine sont loin d'être le lieu idéal pour les soins de santé et la formation médicale. Le gouvernement et les autorités sanitaires de la Centrafrique doivent poursuivre leurs efforts pour renforcer le parc radiologique en équipements lourds et créer une filière de formation des médecins radiologues, des techniciens en radiologie et imagerie médicale, sans oublier le secteur de la maintenance. Cette politique permettra de couvrir les besoins de toute la Centrafrique en termes de personnels de radiologie et de la pérennité des examens de radiologie.

\section{References:}

1. Adeyekun, AA. (2010). Residents' perception of postgraduate radiology training in Nigeria. West Afr J Med:314-7. 
2. Bernard, AM., De Certaines, J. \& Lejeune, JJ. (1987). Abrégé de Résonance Magnétique Nucléaire, collection Abrégés de Médecine, Masson, Paris.

3. Bosembo Ilondjo, M. (2005). Évaluation des besoins et des ressources hospitalières en Afrique centrale (Mémoire). Montréal : Université de Montréal:157p.

4. Comité National de Lutte contre le Cancer (2007). Le cancer du sein in Guide d'information. Ministère de la Santé Publique. Yaoundé. Cameroun. $1^{\text {ère }}$ édition.:37-43.

5. Delaloe, JF., Meuwly, J. \& Ducros, C. (2012). Le dépistage du cancer du sein. Forum Med Suisse :218-220.

6. Etard, C. (2010). Les obligations du radiologue pour la radioprotection du patient. J. Radiol: 1207-11.

7. Fédération Nationale des Médecins Radiologues (2017). Oui à une nouvelle imagerie médicale. Disponible sur le site http ;//fnmr.org/publication/autre/livre/201710-fnmr-projet-nouelle imagerie/files/basic-htlm/page1.html.

8. Gbande, P., Sonhae, L., Adambounou, K., Lamdon, K., N'timon, B. \& Dagbe $\mathrm{M}$ et al. (2017). Analyse du rebut des cliches radiographiques dans le service de radiologie et imagerie médicale du CHU Campus de Lomé au Togo. European Scientific Journal. Novembre:244-253.

9. Guidah, S., Sanussi, S., Abas, B.A. \& Niang, EH. (2012). Apport de l'imagerie médicale dans le bilan des traumatismes crâniens au Niger : à propos de 311 cas à l'Hôpital National de Niamey. J Afr Imag Méd.:4-11.

10. IAEA (2001). Radiological protection for medical exposure to ionizing radiation. Safety standards series ${ }^{\circ}{ }^{\circ}$ s-g- 1.5 . IAEA, Vienna ; 75p.

11. Kaya, G.G., Makita Bangamboula, C., Peko, J.F., Silou, J.F., Manvouri, L. \& Layba I M et al. (2006). Profil des affections orbitaires des affections au Centre Hospitalier et Universitaire de Brazzaville, Congo. Etude rétrospective de 704 cas. J Fr. Ophtalmol.: 281-288.

12. Kouna, N.P., Millogo, A., Siéméfo Kamgang, F.P. \& Assengone-Zeh, Y. (2007). Aspects épidémiologiques et évolutifs des accidents vasculaires au centre hospitalier de Libreville (Gabon). African Journal of neurological Sciences:12-17.

13. National Council on Radiation Protection and Measurements (2009). Ionizing radiation exposure of the population of the United States. NCRP report n॰ 160.

14. Ngou-Mve-Ngouj, P., Mayi-Tsonga, S., Diallo Owono, F.K., Ngo'o Nze, S. \& Ondo N'dong, F. (2009). Lésions infracliniques du sein à Libreville(GABON) : Etude rétrospective de 174 cas. Clin mother Child $\mathrm{n}^{\circ} 1: 1003-1006$. 
15. Nko'o, S.A., Nkam, M., Biwole, S. \& Ongolo-Zogo, P. (2009). Accessibilité des examens d'imagerie médicale et pérennité des équipements lourds au Cameroun. Journal de Radiologie:14-92.

16. Ongolo-Zogo, P., Nguehouo, M.B., Yomi, J. \& Nko'o Amven, S. (2013). Connaissances en matière de radioprotection : enquête auprès des personnels des services hospitaliers radiodiagnostic, radiothérapie et médecine nucléaire à Yaoundé Cameroun. Radioprotection:39-49.

17. Pérusse, C. \& Côté, Z.M-F (1999). Implantation d'un système PACS dans les services d'imagerie médicale de la région des Laurentides : Etude de faisabilité.

18. Tchaou, M., Houadjeto, LCD., Sonhaye, L., Agoda-Koussema, L.K., \& Adlenou N'daema, K. (2018). Etat des lieux et évaluation des formations en Radiologie dans un pays en développement, cas du Togo. European Scientific Journal février :269-278.

19. Werner, J.F., Diakhaté, I., Badji, E., Soko, T.S., Ndiaye, A.R. \& Mbengue et al. (2010). L'imagerie par résonance magnétique haut champ en Afrique Sub saharienne une expérience sénégalaise. J Afr Imag Méd:222-247. 